\title{
Simulation of feedback control system for NTM stabilisation in ASDEX Upgrade
}

\author{
Christopher Rapson ${ }^{\mathrm{a}}$, Francesco Monaco ${ }^{\mathrm{a}}$, Matthias Reich ${ }^{\mathrm{a}}$, Joerg Stober ${ }^{\mathrm{a}}$, Wolfgang Treutterer ${ }^{\mathrm{a}}$, the ASDEX \\ Upgrade Team ${ }^{\mathrm{a}}$ \\ ${ }^{a}$ Max Planck Institute for Plasma Physics, EURATOM Association, Boltzmannstrasse 2, 85748 Garching, Germany
}

\begin{abstract}
Neoclassical Tearing Modes (NTMs) are a class of MHD instability in high beta tokamak plasmas which significantly increase radial transport, thus capping the performance of fusion plasmas. More importantly, NTMs can lead to disruptions which compromise the lifetime of structural components. Several tokamaks have demonstrated that electron cyclotron resonant heating $(\mathrm{ECRH})$ can stabilise NTMs if the power deposition is aligned with the mode location. The deposition location depends on the toroidal magnetic field, flux and density profiles, and can be controlled by tilting the mirror in the ECRH launcher. Until recently, the mirror angle was set by feedforward control at ASDEX Upgrade.

In order to adapt automatically to different discharge scenarios, the system at ASDEX Upgrade has been extended to steer the mirror using feedback control. The mirror must react on the current diffusion time scale, on the order of $100 \mathrm{~ms}$. This is within the capabilities of the mechanical subsystem and real-time plasma diagnostics, but requires careful interfacing between these components. For example, asynchronous data transfer and non-linearities make it difficult to design an analytically optimal controller. Therefore a simulation has been used to test and tune different controller architectures. This simulation is the subject of the current contribution. Performing the optimisation process offline saves valuable experiment time and allows risk-free experimentation with novel designs. Settings which were optimised in the simulation led to considerable improvement of the system performance.
\end{abstract}

Keywords: NTM, neoclassical tearing mode, feedback control, ASDEX Upgrade, simulation

\section{Introduction}

Neoclassical Tearing Modes (NTMs) degrade confinement in tokamaks [1]. Worse still, NTMs can lead to disruptions which compromise the lifetime of structural components [2]. Several methods to mitigate NTMs have been investigated [3], of which localised Electron Cyclotron Resonance Heating (ECRH) is the preferred method to stabilise an NTM. The ECRH deposition location is controlled by moving a mirror in the ECRH launcher, but it is also affected by the plasma density profile, flux profile and toroidal magnetic field. A feedback system is necessary to adjust the mirror position if these parameters - or the position of rational flux surface where the NTM is located - change from their expected values. The mirror control timescale is dictated by flux surface movement. This in turn depends on current diffusion, which occurs on the order of $100 \mathrm{~ms}$ for ASDEX

Email address: chris.rapson@ipp.mpg.de (Christopher Rapson)

Preprint submitted to SOFT 2012 Conference Proceedings
Upgrade. The feedback system will be described only briefly here, from the point of view of the controller. For a full description, please refer to [4]. Real-time diagnostics provide measurements of the position of the mirror actuator $x_{p o l}$ and relevant plasma parameters: the magnetic equilibrium, density profile, magnetic fluctuations and temperature fluctuations. These measurements are used to calculate secondary variables - the island location $\rho_{N T M}$, the location $\rho_{E C R H}$ where ECRH would be deposited given the existing conditions and the sensitivity of this location to a small change in the mirror position $\frac{\partial \rho_{E C R H}}{\partial x_{p o l}}$. $\rho_{\text {target }}$ can be set to follow either a user-defined trajectory, or $\rho_{N T M}$ plus a configurable offset to adjust for asymmetric island shapes and possible modelling errors. The controller repositions the mirror to minimise the error $\rho_{\text {target }}-\rho_{E C R H}$. In this paper, $\rho$ refers to $\rho_{\text {poloidal }}$, the normalised radial magnetic flux co-ordinate. The term ECRH is used whether the microwave beam is used for pure heating or also current drive (ECCD), i.e. whether the toroidal launch angle is 


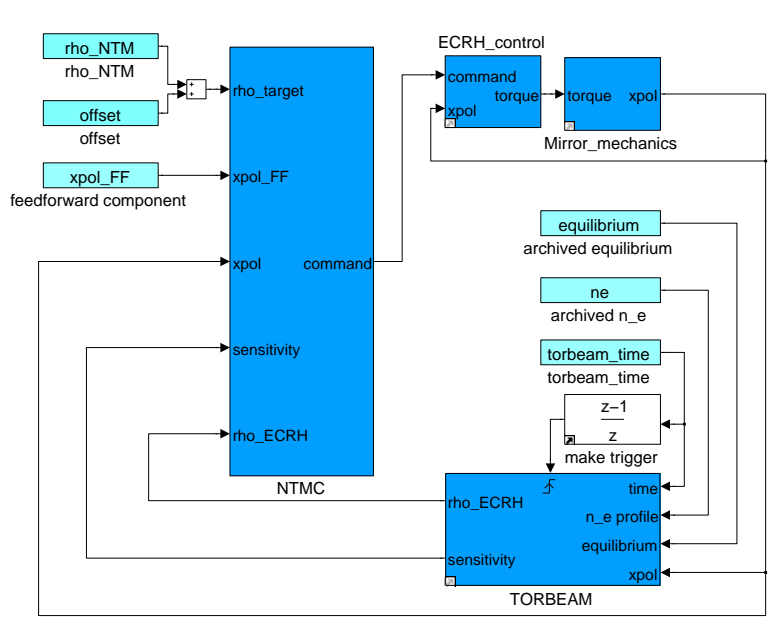

Figure 1: Block diagram of the simulation showing the four main components of the feedback loop and inputs taken from the shotfile archive.

perpendicular to the magnetic field or not. For the controller, only the poloidal launch angle is relevant.

The controller has been optimised using a simulation which is described in Section 2. Section 3 shows how the controller was optimised, and the experimental results are presented in Section 4.

\section{Modelling the Control Loop}

The system was simulated in SIMULINK ${ }^{\circledR}$, with the main components shown in the block diagram in Fig. 1. Several parameters are loaded from shotfile archives, to provide realistic plasma dynamics, or for analysing the system behaviour of previous shots. Alternatively, any parameter can be synthesised. The central NTM controller (NTMC), takes the target position $\rho_{\text {target }}$ as an input and produces a command for the ECRH system. The command is processed by a local controller which drives the mechanical system. Based on the simulated mirror position and archived plasma parameters, a ray tracing code TORBEAM [5] calculates $\rho_{E C R H}$ and $\frac{\partial \rho_{E C R H}}{\partial x_{p o l}}$ which are passed to the NTMC to complete the loop.

TORBEAM is the slowest part of the system. Cycle times are now less than $20 \mathrm{~ms}$, but not deterministic. TORBEAM relies on the real time density profile and equilibrium reconstructions, which have their own latencies (ca. 2 and $6 \mathrm{~ms}$ respectively). Therefore TORBEAM output data can be up to $26 \mathrm{~ms}$ out of date. The simulation calls the same library as the online routine, but the input data may be slightly shifted compared to what was used online. This is because the inbuilt data

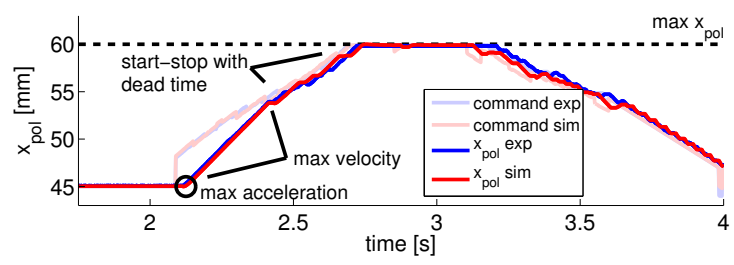

Figure 2: Movement of the linear actuator which drives the mirror launcher angle during shot \#27782. Experimental data are in blue and simulation results in red. Commands from the NTMC are in lighter shades and $x_{p o l}$ lines are in bright colours. Several nonlinear properties of the actuator are marked on the figure.

access routines are not aware of network latencies in the online system. This makes only a small difference to an individual result, but in certain cases it becomes noticeable due to nonlinearities in the feedback loop. See for example Fig. 2 at $t=3.1 \mathrm{~s}$, where the systems de-saturates earlier in the simulation than in the experiment, and maintains a lower $x_{p o l}$ value until $t=3.7 \mathrm{~s}$. More importantly, it was found that ELMs appear as high frequency noise in the equilibrium, which carries through to an uncertainty of \pm 0.02 for the calculation of $\rho_{E C R H}$. These fluctuations can be seen in Fig. 3 .

The NTMC is part of the Discharge Control System at ASDEX Upgrade, and as such produces a new command every $1.5 \mathrm{~ms}$, using the most recent available TORBEAM result. Through consultation with both the source code and the developers, this part of the simulation is an exact replica of the NTMC algorithm. It includes features such as an anti-windup ProportionalIntegral (PI) controller which scales the input error $\rho_{\text {target }}-\rho_{E C R H}$ based on the system sensitivity $\frac{\partial \rho_{E C R H}}{\partial x_{\text {pol }}}$. A feedforward value is added to the output of the PI controller, and the result is quantised to avoid trivially small mirror movements. If any of its inputs become invalid, NTMC switches to a fallback mode, which requires no inputs and is safe by design. It returns to normal functionality if all inputs are valid again. This functionality is provided as part of a standard set of controllers available within the ASDEX Upgrade control system.

The ECRH local controller performs safety checks on new commands, which require three $6 \mathrm{~ms}$ cycles, imposing a dead time of up to $24 \mathrm{~ms}$. After passing the safety checks, the command becomes a reference for a nested position-velocity controller. A current controller uses this output to drive the motor torque. The gains and sample times for these three PI controllers are known, but other details are proprietary and must be estimated by empirically fitting to measurements. For example, it is often observed that the controllers' outputs satu- 
rate and from this can be inferred that the integral components are protected against windup. In shot \#27782, shown in Fig 2, all of these non-linearities are visible. The figure shows that for a large change in command, the response is limited by the maximum velocity set in software. For a slow ramp, the mirror moves in a series of small steps and the response is limited by the dead time between each step. Independent of the mirror motion, discrete changes in command occur for every new evaluation of TORBEAM, in this case every $50 \mathrm{~ms}$.

The mechanical system is modelled as a second order damped system: $m \ddot{x}_{p o l}+c \dot{x}_{p o l}=F=\frac{\text { torque }}{\text { const. }}$. Accurate values for the mass $m$ and damping co-efficient $c$ are not available, so these values have been fitted empirically. With maximum acceleration $\frac{F_{\max }}{m}=100 \mathrm{~ms}^{-2}$ and normalised damping $\frac{c}{m}=750 \mathrm{~s}^{-1}$ the mechanical dynamics are not a limiting factor.

\section{Optimising the Controller}

Initially, values for $K_{p}$ and $K_{i}$ were estimated from a simple model including time constants and delays. However the complexity of the system could not be represented in a simple calculation, and the performance using these estimates was poor. See \#27777 in Fig. 3. Once the complex dynamics were modelled accurately, as described above, the NTMC controller was optimised in a series of simulations. A trajectory with steps in both directions was chosen as the worst case scenario, corresponding to the situation where an island is detected a long way from the pre-step deposition location. Performace was evaluated as a weighted cost function including steady state error, settling time and overshoot. The result is strongly affected by high frequency noise carried through from the magnetic equilibrium, so after the simluation is complete, a moving average filter is used to allow a better assessment of the mirror motion. Separate tests were made to assess robustness, where white noise was added to $x_{p o l}$ and changes were made to the ECRH controller and mechanical system model. Starting with a pure proportional controller, and increasing the gain $K_{p}$, it was found that the system becomes unstable at a low value of $K_{p}=0.9$, even without noise. For all values of $K_{p}$, the steady state error is not acceptable, so $K_{p}$ was reduced to a conservative value and the integral gain $K_{i}$ was increased. The final parameters chosen were $K_{p}=0.2$ and $K_{i}=6.0$, which gives $2 \%$ overshoot (less than the noise), $320 \mathrm{~ms}$ settling time and zero steady state error. The $K_{i}$ value is much larger than initial estimates, and wouldn't have been attempted on the real system without the results from the simulation. Large $K_{i}$ tends to reduce the phase margin and increases the risk of limit cycles in systems with significant latencies. However the simulation showed that such a large value is necessary to minimise the settling time, and is limited by overshoot, not instability. It is also counterintuitive that the loop is more stable for low $K_{p}$ and high $K_{i}$ than vice-versa. Possibly this is related to the low pass filtering effect of the integrator, but this issue remains to be resolved. Derivative gain was not used as the simulation showed an increase in susceptibility to measurement noise and 'derivative kick' problems when switching controller modes. In addition to optimising these gains, the quantisation was removed, since it was discovered that one of the safety checks within the ECRH local controller effectively duplicated this.

In addition to the PI controller, two other controller architectures were simulated. In one configuration, TORBEAM is called 10 times with different values of $x_{p o l}$, and a quartic polynomial is fitted to the function $\rho_{E C R H}\left(x_{p o l}\right)$. The polynomial is solved for $x_{p o l}$ to optimally align the ECRH deposition with the island. If there are multiple solutions, one is chosen that requires the smallest actuator movement. This is computationally more expensive, but reduces settling time to $150 \mathrm{~ms}$. Overshoot is $14 \%$ and there is no steady state error.

A third configuration inverts a linear approximation to $\rho_{E C R H}\left(x_{p o l}\right)$, much like the standard PI controller.

$$
\Delta x \approx \frac{\partial x_{p o l}}{\partial \rho_{E C R H}} \Delta \rho_{E C R H}
$$

So a measured error in $\rho$ can be converted to an error in $x_{p o l}$ and added to the current position.

$$
\begin{aligned}
\text { command } & =x_{\text {pol,meas }} \\
& +K_{p} \frac{\partial x_{p o l}}{\partial \rho_{E C R H}}\left(\rho_{E C R H, \text { target }}-\rho_{E C R H, \text { meas }}\right)
\end{aligned}
$$

$K_{p}=1$ for a pure inversion of $\rho\left(x_{p o l}\right)$, but due to latencies the system becomes unstable, so $K_{p}=0.5$ was chosen. This configuration gives $16 \%$ overshoot, $130 \mathrm{~ms}$ settling time and zero steady state error, and so performs just as well as the nonlinear inversion.

The simulation showed in general that dead time in the system is critical, causing not just a direct time penalty but also affecting system stability and therefore restricting controller gain values. Relatively large overshoot in the inversion-based controllers is a side effect of their fast response to noisy measurements. Since it appears regardless of transients (i.e. steps) in the reference trajectory, "oscillation amplitude" may be a better term than overshoot. Regardless of the term, this behaviour is undesirable. Therefore the PI controller performance is considered superior, despite the slower 

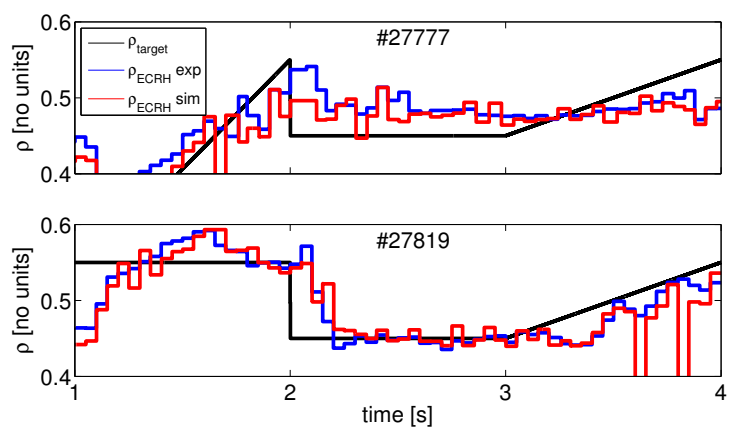

Figure 3: Comparison of system performance with different controller gains. Black shows the reference trajectory or target. Blue line shows experimental results and the red line simulation results. \#27777: $K_{p}=$ 0.3, $K_{i}=0.6$. \#27819: $K_{p}=0.2, K_{i}=6.0$.

response. In addition, the PI controller has many advantages in implementation, being able to draw on standard library functions of the central control system.

\section{Experimental Results}

The optimised parameters from the simulation led to a considerable improvement in the online system performance, as can be seen in Fig. 3. With parameters chosen from initial analytical calculations the system barely responds to step or ramp inputs on timescales of one second in \#27777. In \#27819 the deposition location follows the prescribed trajectory very well, with $300 \mathrm{~ms}$ settling time and overshoot of 0.02 in $\rho$, not dissimilar from the noise level.

These settings were successfully applied to partially stabilise NTMs using one gyrotron [6]. For the results shown in Fig. 3 the target was defined in advance, while stabilisation experiments used the NTM location, evaluated in real time. It is not unusual that the NTM localisation fails - in the best case, this occurs when the mode is stabilised and the amplitude diminishes. Then, the controller changes to its fallback mode. The standard fallback mode used a constant command value which was set before the discharge. However, this can be a large distance from the current mirror position, leading to sudden movements and large stresses on the mirror mechanics. After conducting simulations, a new fallback scheme was implemented where the command is frozen when the inputs become invalid.

Further experiments showed poor tracking performance when the PI controller resumed control. Generally, integrators start from 0 , but here it was found that performance improves if the integrator is initialised such that the first command using the PI controller matches the previous command.
Experiments for Collective Thomson Scattering required a relatively complicated mirror positioning, coordinated between two mirrors. Since all components of the online system are parallelised, simulating multiple mirrors simply requires repeating the simulation. Using the simulation to predict the mirror dynamics allowed the trajectories to be optimised offline, saving several shots which were planned for calibration.

\section{Conclusions}

A simulation of the mirror control feedback loop for NTM stabilisation has been created. The simulation was used to optimise the controller parameters offline, saving experiment time and minimising the stresses on hardware components. With the optimised parameters, the settling time is reduced to $300 \mathrm{~ms}$ for large step changes in the target with minimal overshoot, which was sufficient to partially stabilise NTMs using one gyrotron. The simulation is capable of modelling offnormal events such as diagnostic failures, which is important for simulations of fusion experiments, or experimental devices in general. Because of these capabilities, it continues to be useful for diagnosing issues in previous shots or for planning new experiments.

In the future, the simulation will be used to prepare experiments where the gyrotron power is also controlled from the central controller, and others on pre-emptive control [2]. It is intended that more intelligence will be integrated into the controller, allowing simpler usage by an experiment leader. These advances too will be simulated before their implementation.

\section{References}

[1] T. C. Hender, et al., Chapter 3: MHD stability, operational limits and disruptions, Nucl. Fusion 47 (6) (2007) S128-S202. doi:10.1088/0029-5515/47/6/S03.

[2] B. Esposito, et al., Disruption control on FTU and ASDEX upgrade with ECRH, Nucl. Fusion 49 (6). doi:10.1088/00295515/49/6/065014.

[3] M. Maraschek, Control of neoclassical tearing modes, Nucl. Fusion 52 (7) (2012) 074007.

URL http: //stacks. iop.org/0029-5515/52/i=7/a=074007

[4] M. Reich, et al., ECCD-based NTM control at ASDEX Upgrade, in: Europhysics Conference Abstracts, Proceedings of the 39th EPS Plasma Physics Conference, Stockholm (2012) Paper P1.076, 2012.

URL http://ocs . ciemat.es/epsicpp2012pap/pdf/P1.076.pdf

[5] E. Poli, A. Peeters, G. Pereverzev, TORBEAM, a beam tracing code for electron-cyclotron waves in tokamak plasmas, Comput. Phys. Commun. 136 (1-2) (2001) 90-104. doi:10.1016/S00104655(01)00146-1.

[6] M. Reich, et al., First results of closed loop feedback control of NTMs at ASDEX Upgrade, in: Europhysics Conference Abstracts, Proceedings of the 39th EPS Plasma Physics Conference, Stockholm (2012) Paper PD4.004, 2012. 